\title{
Quelques Nouvelles Propriétés de Régularité de l'Opérateur de Gribov
}

\author{
Marie-Thérèse Aimar ${ }^{1}$, Abdelkader Intissar ${ }^{2}$, Jean-Martin Paoli \\ ${ }^{1}$ Département de Mathématiques, Université de Provence, Place V. Hugo, F-13331 Marseille \\ cedex 3, France \\ 2 Equipe d'analyse spectrale U.R.A. CNRS, $n^{\circ}$ 2053, Département de Mathématiques Université \\ de Corte, Quartier Grossetti, F-20250 Corte, France
}

Received: 28 March 1994/in revised form: 6 December 1994

\begin{abstract}
In this work, we establish new regularity properties for Gribov's operator: $H=\mu A^{*} A_{+} i \lambda A^{*}\left(A+A^{*}\right) A ;(\mu, \lambda) \in \mathbb{R}^{2}$, where $A^{*}$ and $A$ are the creation and annihilation operators. Particularly, we prove that for all $\varepsilon>0, H^{-1}$ is in the class of Carleman's operator $l_{1+\varepsilon}$.
\end{abstract}

\section{Introduction}

Soit $\mathrm{E}$ un espace de Hilbert sur $\mathbb{C}$ muni d'un produit scalaire $\langle$,$\rangle et de la norme$ \|I $\|$ et $K$ un opérateur linéaire compact.

Définition 1. Un opérateur compact $K$ est dit de classe $l_{p}$ de Carleman si la série: $\sum_{n=1}^{\infty}\left[s_{n}\left(\sqrt{K^{*} K}\right)\right]^{p}$ converge où $s_{n}\left(\sqrt{K^{*} K}\right), n=1,2, \ldots$ désigne la suite des valeurs propres de l'opérateur compact hermitien positif $\sqrt{K^{*} K}$.

Remarque 1. [6].

1) Pour une étude des espaces $l_{p}$, on pourra consulter le livre de Gohberg-Krein

2) La théorie des champs de reggeons a été inventée par Gribov [5] en 1967 afin de décrire le comportement à haute énergie des sections efficaces de collisions de particules élémentaires. Elle est caractérisée par l'opérateur de Gribov $H_{\lambda}^{\prime}$ s'exprimant en fonction des opérateurs de création et d'annihilation usuels et agissant sur l'espace de Bargmann [4]:

$$
E=\left\{\varphi: \mathbb{C}^{n} \longrightarrow \mathbb{C} \text { analytiques } ; \int_{\mathbb{C}^{n}} e^{-|z|^{2}}|\varphi(z)|^{2} d z d \bar{z}<\infty \text { et } \varphi(0)=0\right\} .
$$

L'opérateur non auto-adjoint $H_{\lambda}^{\prime}$ est défini par:

$$
H_{\lambda}^{\prime}=\lambda^{\prime} \sum_{j=1}^{n} A_{j}^{* 2} A_{j}^{2}+\mu \sum_{j=1}^{n} A_{j}^{*} A_{j}+i \lambda \sum_{j=1}^{n} A_{j}^{*}\left(A_{j}+A_{j}^{*}\right) A_{j}+\alpha \sum_{j=1}^{n-1}\left(A_{j+1} A_{j}^{*}+A_{j+1}^{*} A_{j}\right),
$$

où $A_{j}^{*}$ et $A_{j}$ désignent respectivement les opérateurs de création et d'annihilation, $\left(\lambda^{\prime}, \mu, \lambda, \alpha\right)$ sont des paramètres réels et $i^{2}=-1$. 
Une étude spectrale complète de $H_{\lambda}^{\prime}$ est donnée dans $[3,7,8$ et 9] et pour $\lambda^{\prime} \neq 0$, la densité du système de ses vecteurs propres généralisés est donnée dans [1 ou 2]. Pour $\lambda^{\prime}=0$, la question de la densité du système des vecteurs propres généralisés de $H_{\lambda}^{\prime}$ dans l'espace de Bargmann est encore ouverte. On donne néanmoins dans ce travail quelques propriétés de régularité $(n=1)$ de cet opérateur limite.

A un site, l'opérateur de Gribov se présente sous la forme $H=\mu H_{0}+i \lambda H_{1}$; $i^{2}=-1$, où:

$H_{0}=A^{*} A$ est l'oscillateur harmonique.

$H_{1}=A^{*}\left(A+A^{*}\right) A$ est l'opérateur cubique de la théorie des champs de reggeons $\mu$ est l'intercept de Pomeron.

$\lambda$ est le triple coupling de Pomeron.

Dans la représentation de Bargmann les opérateurs $A^{*}$ et $A$ ne sont autres que la multiplication par $z$ et la dérivation par rapport à $z$. $H$ s'écrit ainsi:

$$
H=H(\mu, \lambda)=i \lambda z \frac{d^{2}}{d z^{2}}+\left(i \lambda z^{2}+\mu z\right) \frac{d}{d z} .
$$

L'adjoint formel de $\mathrm{H}$ est donné par:

$$
H^{T}=H(\mu, \lambda)^{T}=H(\mu,-\lambda) \text { et }\left(H^{T}\right)^{T}=H .
$$

Remarque 2.

i) $e_{k}(z)=\frac{z^{k}}{\sqrt{k !}} ; k=1,2, \ldots$ est une base orthonormée de $E$.

ii) $D(A)=\{\varphi \in E ; A \varphi \in E\}$ s'injecte de façon compacte dans $E$.

iii) Soit $\mathscr{P}$ l'ensemble des polynômes qui s'annulent à l'origine, $\mathscr{P}$ est dense dans $E$.

iv) L'opérateur $H$ de domaine les polynômes $\mathscr{P}$ est fermable.

Lemme 1 (A. Intissar [7]).

i) $\|\varphi\| \leqq\|A \varphi\|$ pour tout $\varphi$ dans $D(A)$.

ii) $\varphi$ appartient à $E$ si et seulement si l'application $z \rightarrow \frac{\varphi^{\prime}(z)-\varphi^{\prime}(0)}{z}$ appartient à $E$.

iii) Si $\varphi \in E$, l'intégrale $\int_{\mathbb{C}} \frac{e^{-|z|^{2}}}{1+|z|^{2}}\left|\varphi^{\prime}(z)\right|^{2} d x d y$ est convergente.

iv) Si $\varphi \in E$, les intégrales $\int_{-\infty}^{+\infty} e^{-y^{2}}|\varphi(x+i y)|^{2} d y$ et $\int_{-\infty}^{+\infty} \frac{e^{-y^{2}}}{1+y^{2}}\left|\varphi^{\prime}(x+i y)\right|^{2} d y$ sont convergentes pour tout $x$.

Dans toute la suite, on pose $H_{\max }(\mu, \lambda) \varphi=H \varphi$ pour $\varphi \in D_{\max }=D(H)=$ $\{\varphi \in E ; H \varphi \in E\}$ et on définit:

* L'extension minimale de $H$ par:

$$
\begin{gathered}
H_{\min }=H_{\min }(\mu, \lambda)=\mu A^{*} A+i \lambda A^{*}\left(A+A^{*}\right) A \text { de domaine: } \\
D_{\min }=\left\{\varphi \in E ; \exists p_{n} \in \mathscr{P} \text { et } \exists \psi \in E ; \lim p_{n}=\varphi\right. \text { et } \\
\left.\lim H p_{n}=\psi=H_{\min } \varphi \text { quand } n \rightarrow \infty\right\}
\end{gathered}
$$

$H_{\text {min }}$ ainsi défini, est la fermeture de la restriction de $H$ à $\mathscr{P}$. Par conséquent $\mathscr{P}$ est le "coeur" de $H_{\min }$ c'est-à-dire: l'ensemble des éléments $\left(p, H_{\min } p\right)$ avec $p \in \mathscr{P}$ est 
dense dans $G\left(H_{\min }\right)$ où $G\left(H_{\min }\right)$ désigne le graphe de $H_{\min }$.

$$
\text { * } H_{\max }=H_{\max }(\mu, \lambda), \quad\left(H^{T}\right)_{\min }=H_{\min }(\mu,-\lambda) \quad \text { et } \quad\left(H^{T}\right)_{\max }=H_{\max }(\mu,-\lambda) \text {. }
$$

Alors on a:

$\left\langle H_{\max }(\mu, \lambda) \varphi, p\right\rangle=\langle H \varphi, p\rangle=\left\langle\varphi, H^{T} p\right\rangle$ pour tout $p \in P$ (ensemble des polynômes), ce qui est équivalent à $\left\langle H_{\max }(\mu, \pm \lambda) \varphi, q\right\rangle=\left\langle\varphi, H_{\min }(\mu, \mp \lambda) q\right\rangle$ pour tout $q \in D_{\min }$ et $\varphi \in D_{\max }$

Il en résulte que

$$
H_{\min }(\mu, \pm \lambda)^{*}=H_{\max }(\mu, \mp \lambda) \text { où } H_{\min }(\mu, \pm \lambda)^{*} \text { est l'adjoint de } H_{\min }(\mu, \pm \lambda) .
$$

Théorème 1. Pour $\mu>0$. Alors

1) L'opérateur $H_{\max }(\mu, \lambda)$ est injectif pour tout $\lambda \in \mathbb{R}$.

2) L'opérateur $H_{\min }(\mu, \lambda)$ est bijectif pour tout $\lambda \in \mathbb{R}$.

3) $H_{\max }(\mu, \lambda)=H_{\min }(\mu, \lambda)$ pour tout $\lambda \in \mathbb{R}$.

\section{Démonstration.}

1) Soit $\varphi \in D(H)$ vérifiant $H \varphi=0$ alors $i \lambda z \varphi^{\prime \prime}(z)+\left(i \lambda z^{2}+\mu z\right) \varphi^{\prime}(z)=0$ et $\varphi^{\prime}(z)=c \operatorname{Exp}\left(\frac{-z^{2}}{2}+i \frac{\mu}{\lambda} z\right)$ où $c$ est une constante. En appliquant par exemple la propriété (iv) du Lemme 1, on vérifie que $\varphi$ n'appartient pas à l'espace de Bargmann $E$.

2) La démonstration de cette propriété repose sur le lemme précédent. En effet, pour $\mu>0, \operatorname{Re}\left\langle H_{\min } \varphi, \varphi\right\rangle=\mu\|A \varphi\|^{2}$ d'où l'on déduit, en appliquant (i) du lemme, que $\|\varphi\| \leqq \frac{1}{\mu}\left\|H_{\min } \varphi\right\|$ pour tout $\varphi \in D_{\min }$. Il en résulte que $H_{\min }$ est injectif et d'image fermée. D'autre part, l'orthogonal de l'image de $H_{\min }$ est $\{0\}$. En effet $\left\langle\psi, H_{\min }(\mu, \lambda) \varphi\right\rangle=0$ pour tout $\varphi \in D_{\min }$ est équivalent à $\langle\psi, H p\rangle=0$ pour tout $p \in \mathscr{P}$ donc $H_{\max }(\mu,-\lambda) \psi=H_{\min }(\mu, \lambda)^{*} \psi=0$, il résulte de la propriété (1) que $\psi=0$.

3) Soit $\varphi \in D_{\max }$, comme $H_{\min }$ est bijectif, alors il existe $q \in D_{\min }$ tel que $H_{\max } \varphi=H_{\min } q$. Comme $D_{\min } \subset D_{\max }$, on en déduit que $H_{\max }(\varphi-q)=0$. Il en résulte (en appliquant l'injectivité de $H_{\max }$ ) que $\varphi=q \in D_{\min }$.

\section{Corollaire.}

1) L'inverse de $H$ est compact.

2) Pour tout $\lambda \in \mathbb{R}$ et $\mu>0$ on $a:\left\|H^{-1} \psi\right\| \leqq \frac{1}{\mu}\|\psi\|$ pour tout $\psi \in E$.

\section{Démonstration.}

1) Il suffit de remarquer d'une part que l'ensemble résolvant de $H_{\min }$ est non vide et d'autre part que $D(H)$ s'injecte dans $D(A)$ de façon continue or ce dernier s'injecte dans $E$ de façon compacte.

2) Comme pour tout $\lambda \in \mathbb{R}$ et $\mu>0$ on a $\mu\|\varphi\| \leqq\|H \varphi\|$ pour tout $\varphi \in D(H)$, il en résulte que $\left\|H^{-1} \psi\right\| \leqq \frac{1}{\mu}\|\psi\|$ pour tout $\psi \in E$.

\section{La Subordination de l'Oscillateur Harmonique à l'Opérateur de Gribov}

Définition 2. Soit $S$ et $B$ deux opérateurs linéaires de domaine respectif $D(S)$ et $D(B)$. On dit que $B$ est p-subordonné $(0 \leqq p \leqq 1)$ à $S$ si:

a) $D(S) \subset D(B)$.

b) Il existe $C>0$ tel que $\|B \varphi\| \leqq C\|S \varphi\|^{p}\|\varphi\|^{1-p}$ pour tout $\varphi$ dans $D(S)$.

Pour $p=1$, on dit que $B$ est subordonné à $S$. 
Soit $H_{0}=A^{*} A$ l'oscillateur harmonique de domaine $D\left(H_{0}\right)=\left\{\varphi \in E ; A^{*}\right.$ $A \varphi \in E\}$

Lemme 2 (Quelques inégalités a priori).

1) $\operatorname{Pour}(\mu, \lambda) \in \mathbb{R}^{2},\left|\operatorname{Re}\left\langle H H_{0}^{-1} p, p\right\rangle\right| \geqq \mu\|p\|^{2}-|\lambda| \cdot\left|\operatorname{Im}\left\langle A H_{0}^{-1} p, p\right\rangle\right| \quad \forall p \in \mathscr{P}$.

2) $\left\|A H_{0}^{-1} p\right\| \leqq\|p\| \quad \forall p \in \mathscr{P}$.

3) $\|A \varphi\| \leqq\left\|H_{0} \varphi\right\|^{1 / 2}\|\varphi\|^{1 / 2} \quad \forall \varphi \in D\left(H_{0}\right)$.

4) Pour $\mu>0, \varepsilon>0$ et $\lambda \neq 0$ on $a$ :

a) $(\mu-\varepsilon)\left\|H_{0} \varphi\right\| \leqq\|H \varphi\|+\frac{|\lambda|^{2}}{4 \varepsilon}\|\varphi\| \quad \forall \varphi \in D(H)$,

b) $(\mu-\varepsilon)\left\|H_{0} H^{-1} \psi\right\| \leqq\left(1+\frac{|\lambda|^{2}}{4 \mu \varepsilon}\right)\|\psi\| \quad \forall \psi \in E$.

\section{Démonstration.}

1) Soit $p \in \mathscr{P}$, en remarquant que $H_{0}^{-1} \mathscr{P}=\mathscr{P}=H_{0} \mathscr{P}$, on peut écrire:

$$
\begin{aligned}
H H_{0}^{-1} p & =\mu p+i \lambda A^{*}\left(A+A^{*}\right) A H_{0}^{-1} p \\
& =\mu p+i \lambda A^{*} A^{2} H_{0}^{-1} p+i \lambda A^{* 2} A H_{0}^{-1} p \\
& =\mu p+i \lambda\left[A A^{*}-I\right] A H_{0}^{-1} p+i \lambda A^{*} p \\
& =\mu p+i \lambda\left(A+A^{*}\right) p-i \lambda A H_{0}^{-1} p .
\end{aligned}
$$

Il en résulte que $\operatorname{Re}\left\langle H H_{0}^{-1} p, p\right\rangle=\mu\|p\|^{2}-\lambda \operatorname{Im}\|p\|^{2}-\lambda \operatorname{Im}\left\langle A H_{0}^{-1} p, p\right\rangle$ et donc $\left|\operatorname{Re}\left\langle H H_{0}^{-1} p, p\right\rangle\right| \geqq \mu\|p\|^{2}-|\lambda| \cdot\left|\operatorname{Im}\left\langle A H_{0}^{-1} p, p\right\rangle\right|$.

2) Comme $A H_{0}^{-1} p=\sum_{k=1}^{N} a_{k} A H_{0}^{-1}\left(\frac{z^{k}}{\sqrt{k !}}\right)=\sum_{k=1}^{N} \frac{a_{k}}{\sqrt{k}}\left(\frac{z^{k-1}}{\sqrt{(k-1) !}}\right)$, on en déduit que:

$$
\left\|A H_{0}^{-1} p\right\|^{2} \leqq\|p\|^{2}
$$

3) Comme $\|A \varphi\|^{2}=\left\langle A^{*} A \varphi, \varphi\right\rangle=\left\langle H_{0} \varphi, \varphi\right\rangle \leqq\left\|H_{0} \varphi\right\| \cdot\|\varphi\|$, on a:

$$
\|A \varphi\| \leqq\left\|H_{0} \varphi\right\|^{1 / 2}\|\varphi\|^{1 / 2} \quad \forall \varphi \in D\left(H_{0}\right) .
$$

4) a) En appliquant (1) de ce lemme on déduit que:

$$
\left\|H H_{0}^{-1} q\right\|\|q\| \geqq \mu\|q\|^{2}-|\lambda| \cdot\left\|A H_{0}^{-1} q\right\| \cdot\|q\| .
$$

Et d'aprés (3) de ce lemme on a:

$$
\left\|A H_{0}^{-1} q\right\| \leqq\|q\|^{1 / 2}\left\|H_{0}^{-1} q\right\|^{1 / 2} \leqq \frac{\varepsilon}{|\lambda|}\|q\|+\frac{|\lambda|}{4 \varepsilon}\left\|H_{0}^{-1} q\right\|, \text { alors }
$$

$\left\|H H_{0}^{-1} q\right\| \geqq(\mu-\varepsilon)\|q\|-\frac{|\lambda|^{2}}{4 \varepsilon}\left\|H_{0}^{-1} q\right\|$ c'est-à-dire:

$$
(\mu-\varepsilon)\|q\| \leqq\left\|H H_{0}^{-1} q\right\|+\frac{|\lambda|^{2}}{4 \varepsilon}\left\|H_{0}^{-1} q\right\| \quad \forall q \in \mathscr{P} .
$$

Comme $H_{0}^{-1} \mathscr{P}=\mathscr{P}$, on obtient:

$$
(\mu-\varepsilon)\left\|H_{0} p\right\| \leqq\|H p\|+\frac{|\lambda|^{2}}{4 \varepsilon}\|p\| \quad \forall p \in \mathscr{P} .
$$


De la densité des polynômes $\mathscr{P}$ dans l'espace de Bargmann $E$ et de l'égalité du domaine minimal de $H$ et de son domaine maximal, on déduit que:

$$
(\mu-\varepsilon)\left\|H_{0} \varphi\right\| \leqq\|H \varphi\|+\frac{|\lambda|^{2}}{4 \varepsilon}\|\varphi\| \quad \forall \varphi \in D(H) .
$$

b) Il suffit de combiner cette dernière inégalité et l'inégalité du corollaire pour obtenir:

$$
(\mu-\varepsilon)\left\|H_{0} H^{-1} \psi\right\| \leqq\left(1+\frac{|\lambda|^{2}}{4 \mu \varepsilon}\right)\|\psi\| \quad \forall \psi \in E .
$$

Ces inégalités a priori nous permettent d'obtenir:

\section{Théorème 2.}

1) $A$ est $\frac{1}{2}$-subordonné à $H_{0}$.

2) Le domaine de $H$ est inclus dans celui de $H_{0}: D(H) \subset D\left(H_{0}\right)$.

3) $H_{0}$ est subordonné à $H$.

\section{Régularité de l'Opérateur de Gribov}

Théorème 3. $\forall \varepsilon>0$, on $a H^{-1} \in l_{1+\varepsilon}$.

Démonstration. De l'inégalité a priori $\frac{\mu}{2}\left\|H_{0} \varphi\right\| \leqq\|H \varphi\|+\frac{|\lambda|^{2}}{2 \mu}\|\varphi\| \quad \forall \varphi \in D(H)$ (Lemme 2 avec $\varepsilon=\frac{\mu}{2}$ ), on déduit que l'opérateur $B=H_{0} H^{-1} \in L(E)$ où $L(E)$ désigne l'espace de tous les opérateurs linéaires bornés de $E$ dans lui même. Par conséquent:

$$
H^{-1}=H_{0}^{-1} B \text { et comme } H_{0}^{-1} \in l_{1+\varepsilon} \text { on en déduit que } H^{-1} \in l_{1+\varepsilon} .
$$

\section{Continuité de $\boldsymbol{H}^{-1}$ par rapport au paramètre $\lambda$}

Proposition. Posons $f(\lambda)=H^{-1}=\left(\mu H_{0}+i \lambda H_{1}\right)^{-1}$ pour $\lambda \in \mathbb{R}$. Alors la fonction $f: \mathbb{R} \longrightarrow L(E)$ est continue.

Démonstration. Pour $\lambda_{0} \neq 0$, on a:

$$
\begin{aligned}
\left(\mu H_{0}+i \lambda H_{1}\right)^{-1}-\left(\mu H_{0}+i \lambda_{0} H_{1}\right)^{-1}= & i\left(\lambda_{0}-\lambda\right)\left(\mu H_{0}+i \lambda H_{1}\right)^{-1} \\
& \times H_{1}\left(\mu H_{0}+i \lambda_{0} H_{1}\right)^{-1},
\end{aligned}
$$

en appliquant le corollaire on en déduit que:

$$
\begin{gathered}
\left\|\left(\mu H_{0}+i \lambda H_{1}\right)^{-1}-\left(\mu H_{0}+i \lambda_{0} H_{1}\right)^{-1}\right\| \leqq \frac{1}{\mu}\left\|H_{1}\left(\mu H_{0}+i \lambda_{0} H_{1}\right)^{-1}\right\| \cdot\left|\lambda_{0}-\lambda\right| \text { et comme } \\
\left\|H_{1}\left(\mu H_{0}+i \lambda_{0} H_{1}\right)^{-1}\right\|=\frac{1}{\left|\lambda_{0}\right|}\left\|\left(\mu H_{0}+i \lambda_{0} H_{1}-\mu H_{0}\right)\left(\mu H_{0}+i \lambda_{0} H_{1}\right)^{-1}\right\| \\
\leqq \frac{1}{\left|\lambda_{0}\right|}\left(1+\mu\left\|H_{0}\left(\mu H_{0}+i \lambda_{0} H_{1}\right)^{-1}\right\|\right) .
\end{gathered}
$$


En appliquant l'inégalité $\frac{\mu}{2}\left\|H_{0} \varphi\right\| \leqq\|H \varphi\|+\frac{|\lambda|^{2}}{2 \mu}\|\varphi\| \forall \varphi \in D(H)$ (Lemme 2 avec $\varepsilon=\frac{\mu}{2}$ ), on obtient:

$$
\frac{1}{\left|\lambda_{0}\right|}\left(1+\mu\left\|H_{0}\left(\mu H_{0}+i \lambda_{0} H_{1}\right)^{-1}\right\|\right) \leqq \frac{1}{\left|\lambda_{0}\right|}\left(3+\frac{\left|\lambda_{0}\right|^{2}}{\mu}\right) .
$$

Par conséquent, on a:

$$
\left\|\left(\mu H_{0}+i \lambda H_{1}\right)^{-1}-\left(\mu H_{0}+i \lambda_{0} H_{1}\right)^{-1}\right\| \leqq \frac{1}{\mu} \frac{1}{\left|\lambda_{0}\right|}\left(3+\frac{\left|\lambda_{0}\right|^{2}}{\mu}\right)\left|\lambda_{0}-\lambda\right| .
$$

Pour $\lambda_{0}=0$, on a:

$$
\left\|\left(\mu H_{0}+i \lambda H_{1}\right)^{-1} \Psi-\frac{1}{\mu} H_{0}^{-1} \Psi\right\| \leqq\left\|\left(\mu H_{0}+i \lambda H_{1}\right)^{-1}\right\|\left\|\Psi-\frac{1}{\mu}\left(\mu H_{0}+i \lambda H_{1}\right) H_{0}^{-1} \Psi\right\|
$$

pour tout $H_{0}^{-1} \Psi \in D(H)$, c'est-à-dire pour tout $\Psi \in H_{0}[D(H)]$. En appliquant à nouveau le corollaire on en déduit que:

$$
\left\|\left(\mu H_{0}+i \lambda H_{1}\right)^{-1} \Psi-\frac{1}{\mu} H_{0}^{-1} \Psi\right\| \leqq \frac{|\lambda|}{\mu^{2}}\left\|H_{1} H_{0}^{-1} \Psi\right\|
$$

qui tend vers 0 lorsque $\lambda$ tend vers 0 pour tout $\Psi \in H_{0}[D(H)]$. Comme $H_{0}[D(H)]$ est dense dans $E$ on en déduit la continuité de la fonction $f(\lambda)$ à l'origine.

Remerciements. L'un des auteurs, le professeur A. Intissar, remercie le professeur H. Araki pour l'intérêt constant qu'il a porté à l'étude de cet opérateur de Gribov.

\section{Bibliographie}

1. Aimar, M.T., Intissar, A., Paoli, J.M.: Densité des vecteurs propres généralisés d'une classe d'opérateurs non auto-adjoints à résolvante compacte. C.R. Acad. Sci. Paris, t. 315, Série I (1992)

2. Aimar, M.T., Intissar, A., Paoli, J.M.: Densité des vecteurs propres généralisés d'une classe d'opérataurs compacts non auto-adjoints et applications. Commun. Math. Phys. 156, 169-177 (1993)

3. Ando, T., Zerner, M.: Sur une valeur propre d'un opérateur. Commun. Math. Phys. 93, 123-139 (1984)

4. Bargmann, V.: On a Hilbert space of analytic functions and an associated integral transform I. Commun. Pure App. Math. 14 (1962)

5. Gribov, V.: J.E.T.P. (Sov. Phys.) 26 (1968)

6. Gohberg, I.C., Krein, M.: Introduction to the theory of linear non-self adjoint operators. 18 Providence R.I: A.M.S., 1969

7. Intissar, A.: Etude spectrale d'une famille d'opérateurs non-symétriques intervenant dans la théorie des champs de reggeons. Commun. Math. Phys. 113, 263-297 (1987)

8. Intissar, A.: Quelques nouvelles propriétés spectrales de l'hamiltonien de la théorie des champs de reggeons. C.R. Acad. Sci. Paris, t. 308, Série I (1989)

9. Intissar, A.: Théorie spectrale dans l'espace de Bargmann. Cours de D.E.A., Université de Besançon (1989) 